\title{
Contribuições para a Educação em Teoria de Marketing: uma Agenda Profissional e Acadêmica
}

\section{Contributions to Marketing Theory Education: a Professional and Academic Agenda}

\author{
Josemar Jeremias Bandeira de Souza \\ Universidade Federal da Paraíba - UFPB - Brasil \\ josemarbandeira@gmail.com \\ ORCID: 0000-0002-3715-7007
}

Submetido em 03/04/2020; Aprovado em 23/07/2020.

\begin{abstract}
Resumo
Objetivo - O objetivo desse artigo é abordar problemas históricos da educação e do desenvolvimento científico do marketing, tais como a oferta de competências amplas para acadêmicos e profissionais de mercado; a fragmentação da disciplina decorrente de sua abrangência quase ilimitada; a alegada crise de identidade decorrente da fragmentação e suas consequências para a formação dos egressos de programas de doutorados com ênfase ou linhas de marketing. Metodologia - trata-se de um estudo bibliográfico que acompanha o surgimento das temáticas no percurso histórico do marketing. Principais resultados - os resultados mostram: 1) a importância da educação em teoria de marketing para acadêmicos e profissionais do mercado; 2) o papel de uma teoria geral de marketing para o reconhecimento da relevância profissional e a cientificidade do marketing; 3) o papel da disciplina Teoria de Marketing ministrada em nível doutoral para a difusão de conhecimentos sedimentares da área como um todo. Contribuições - 0 artigo traz avanços na construção de uma agenda para a formação em teoria de marketing e importantes reflexões sobre a estruturação filosófica, histórica, tecnológica e social da disciplina.

Palavras-chave: Teoria de marketing; formação em marketing, educação abrangente.
\end{abstract}

\section{Abstract}

Goal - The purpose of this article is to address historical problems of education and scientific development of marketing, such as the provision of broad competencies for academics and market professionals; the fragmentation of discipline resulting from its almost unlimited scope; the alleged identity crisis resulting from fragmentation and its consequences for the formation of graduates of doctoral programs with emphasis or marketing lines. Methodology - this is a bibliographic study that follows the emergence of themes in the historical path of marketing. Main results - the results show: 1) the importance of education in marketing theory for academics and market professionals; 2) the role of a general marketing theory for the recognition of professional relevance and the scientificity of marketing; 3 ) the role of the marketing theory discipline taught at the doctoral level for the dissemination of sedimentary knowledge of the area as a whole. Contributions - the article brings advances in the construction of an agenda for training in marketing theory and important reflections on the philosophical, historical, technological and social structuring of the discipline.

Keywords: Marketing theory; marketing education, broad education.

\section{Introdução}

As habilidades e competências necessárias para suprir as carências acadêmicas e de mercado aparecem como um grande dilema em textos sobre formação e treinamento em marketing. Esse assunto ganhou espaço com o emblemático artigo de Converse (1945), que questionava se o marketing era arte ou ciência, abrindo o caminho para reflexões sobre as diferenças entre as práticas gerenciais e acadêmicas da área. Depois dele, muitos estudiosos se envolveram nessa discussão. Alguns chegaram a propor a criação de um vocabulário que distinguisse um campo do outro, clarificando as diferenças entre o mercado e a academia (Calder et al., 1981; Bringberg \& Mcgrath, 1983). Em oposição a esse caminho, Brown (2001) argumentava que tal distanciamento traria prejuízos para a disciplina. Mais recentemente, outros teóricos seguiram a perspectiva dele, considerando que a estratificação do conhecimento não seria 
um bom caminho e que os estudantes de marketing deveriam ter uma formação mais ampla, capaz de atender aos dois campos (Honea \& Castro; Peter, 2017; Ye et al., 2017; Rapsold \& Hemais, 2018).

Não obstante, 0 aprofundamento da discussão também ampliou a gama de perspectivas em torno do problema. Hunt, Chonko e Wood (1986) investigaram a eficiência da formação em marketing, considerando o efeito exercido pela diplomação sobre as posições ocupadas no mercado pelos egressos dos vários níveis de graduação e pós-graduação ofertados pela academia. A conclusão do estudo foi que a formação em marketing não havia sido relevante para os cargos ocupados no mundo profissional. Recentemente, Bacon (2017) publicou uma revisão desse estudo, concluindo, de forma análoga aos primeiros autores, que não há necessariamente uma relação entre a diplomação em marketing e o sucesso profissional. As conclusões desses dois estudos lançam dúvidas sobre a eficiência da educação em marketing no que tange à oferta de conhecimentos suficientemente abrangentes para atender às demandas acadêmicas e de mercado.

Outra perspectiva é apresentada por Smith et al. (2015), ao afirmar que acadêmicos e praticantes de marketing figuram em ambientes epistemologicamente distintos. Segundo esses autores, os acadêmicos estão sujeitos a um ambiente mais homogêneo, restrito ao domínio da lógica racional e do rigor científico. Logo, envolvidos em uma lógica dogmaticamente cartesiana. Por outro lado, os praticantes exercem suas atividades em um campo mais heterogêneo, segmentado, fragmentado, frequentemente recorrendo ao improviso. Nesse ambiente, as suposições cartesianas muitas vezes são impraticáveis. Partindo desses dois cenários tão diametralmente opostos, os autores concluem que o afastamento entre as estratégias acadêmicas e as práticas de mercado se deve à herança e internalização dessas suposições cartesianas, que são persistentemente dominantes no mundo acadêmico e impraticáveis no mundo prático.

Uma terceira perspectiva é defendida por Kumar (2017). Mesmo reconhecendo que o marketing profissional tem outro ritmo de trabalho e é movido por outras forças que não apenas o conhecimento, principalmente naquilo que diz respeito à tomada de decisões, Kumar (2017) resume o problema do distanciamento aos efeitos da metodologia de ensino. Ele defende que o processo de integração entre formação acadêmica e as práticas de mercado já existe e é eficiente. Assim, a academia necessitaria observar quais são as dificuldades e similaridades entre os dois campos, bem como os elementos essenciais para se estabelecer os limites do marketing científico. Sua proposta é que haja uma integração das forças teóricas com as necessidades de mercado em uma metodologia baseada em conhecimento teórico e posterior aplicação, que ele chama de "Perpetuidade do Ciclo Teoria-Prática-Teoria".

Neste artigo, abordamos problemas históricos da educação e do desenvolvimento científico do marketing, buscando compreender como a formação dos egressos das linhas de marketing dos programas de doutorado em administração é afetada por questões como a busca por competências abrangentes; a fragmentação da disciplina, decorrente das abordagens quase ilimitadas; a alegada crise de identidade decorrente da fragmentação. Argumentamos, então, que a disciplina Teoria de Marketing ofertada nos programas de pós-graduação em Administração tem papel crucial no contexto geral da educação de marketing.

O objetivo do presente trabalho é propor um conjunto sedimentar de conhecimentos de teoria de marketing que ofereça aos egressos das linhas de marketing dos programas de doutorado em administração uma formação generalista capaz de contribuir profissional e academicamente com a difusão e/ou aplicação do marketing e suas ferramentas aos múltiplos contextos. Para tanto, argumentamos que as linhas de marketing dos programas de doutorado em administração exercem papel preponderante na cadeia de formação da área, cabendo a elas a tarefa de ofertar uma instrumentalização ampla capaz de mitigar problemas teóricos e práticos que tangenciam o marketing como um todo. Defendemos que a discussão aqui realizada, juntamente com o conjunto de conhecimentos propostos, fecha uma importante lacuna da formação em marketing: a fragmentação do conhecimento e suas consequências para os campos científico e profissional.

\section{Abrangência da educação em teoria de marketing e a formação de competências}

Na linha da perspectiva apresentada por Kumar (2017), dois números especiais do Journal of Marketing Education (v. 39, n. 2 e n. 3, 2017) buscaram lançar luzes sobre os meios de aproximar academia e mercado através de transformações no ensino em marketing. As discussões, no entanto, foram restritas ao nível de graduação e às questões de ordem metodológica, sem considerar as necessidades de formação nos níveis de pós-graduação e de revisão dos conteúdos. Questões mais amplas da teorização, 
tais como identidade e natureza do marketing, fragmentação do conhecimento e sistemas em que as trocas não são financeiras (Layton, 2007; El-Ansary, Shaw; \& Lazer, 2018; Haase, Backer \& Pick, 2018) requerem, também, competências profissionais distintas, tanto na academia quanto no mercado, mas foram ignoradas em todos os artigos.

A perspectiva de macromarketing - principalmente, no ponto que nos faz entender o marketing como uma função que pode ser aplicada aos inúmeros contextos das relações de troca (Kotler \& Levy, 1969; Bagozzi, 1975; Layton, 2007) - também exige novas competências vinculadas a um conjunto de conteúdos muito mais amplo, incluindo, por exemplo, marketing social e marketing público (Costa, 2015), questões de aplicação como o bem público (Kadirov, 2018), soluções para problemas macrossociais (Kennedy, 2016), ética (Murphy, 2017), justiça distributiva (Ferrell \& Ferrell, 2008), cocriação de valor (Rajan \& Read, 2016), desenvolvimento regional (Layton, 1985) etc.

Essa gama de assuntos não exaure as possibilidades de aplicação das ferramentas de marketing, mas é suficiente para sustentar o argumento de que a formação na área precisa ter uma larga abrangência em termos de conteúdo e que os esforços metodológicos, apesar das crenças de Kumar (2017), não cobrem todas essas necessidades. Na Figura 1, ilustramos esse primeiro conjunto de problemas relacionados à formação de competências em função da disciplina Teoria de Marketing, considerando três campos de atuação e duas esferas de abrangência.

Os três campos considerados foram: 1) o campo formal do conhecimento, ou simplesmente campo acadêmico, no qual a teoria de marketing é mais amplamente desenvolvida e analisada; 2) o campo de interpretação do conhecimento, que se refere a acadêmicos e praticantes que interpretam o conhecimento em níveis de alta gerência, consultorias e pesquisas aplicadas; e 3) o campo da aplicação do conhecimento, considerando os profissionais de marketing que atuam em cargos operacionais das inúmeras áreas possíveis.

Embora as reivindicações de aproximação estejam normalmente restritas aos campos gerencial ${ }^{\mathrm{i}}$ e acadêmico, sem distinções internas (Brown, 2001; Ewing \& Ewing, 2017; Kumar, 2017; Rapsold \& Hemais, 2018), consideramos que uma divisão intermediária, referente aos profissionais e acadêmicos que atuam como intérpretes do marketing científico para o marketing gerencial, seria necessária para compreender as necessidades de formação teórica e para atender às vindicações de macromarketing (Layton, 2007; Kennedy, 2016; Haase, Becker \& Pick, 2018; Kadirov, 2018). Dessa forma, a disciplina Teoria de Marketing estaria posicionada, em relação à distribuição do conhecimento nas áreas acadêmica e gerencial, de acordo com o modelo da Figura 1.

Figura 1 - Posicionamento da disciplina de Teoria de Marketing em relação à dicotomia dos campos tecnológico e acadêmico.

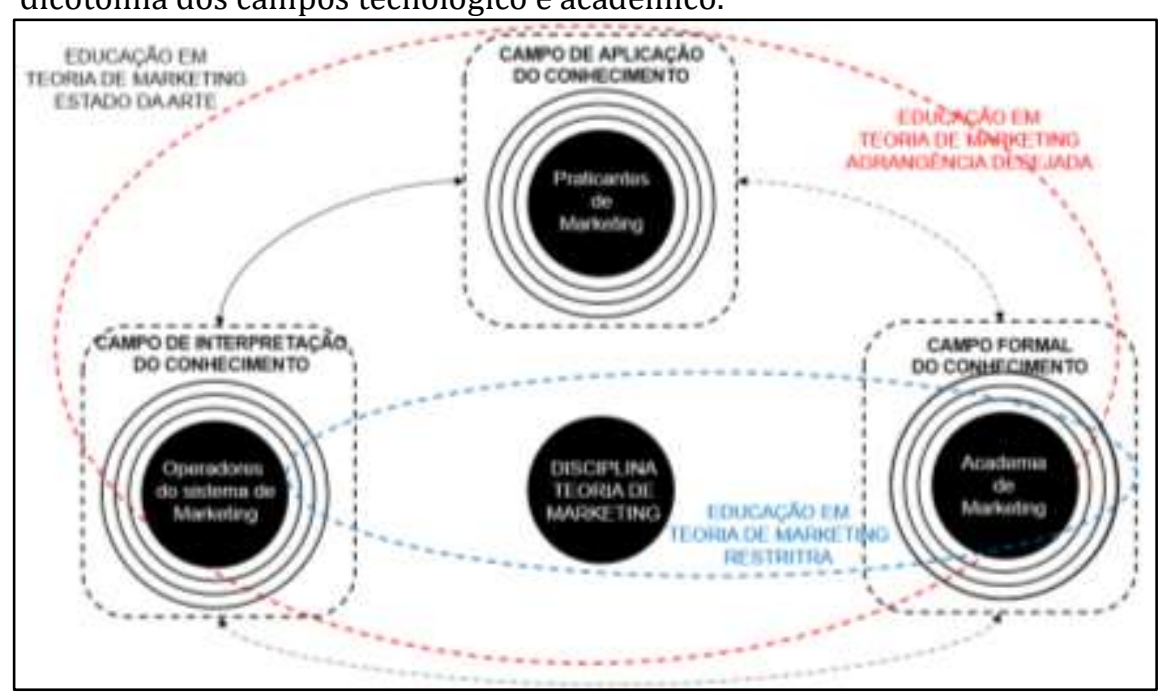

Fonte: Elaboração própria (2020).

A Figura 1 também apresenta duas esferas de abrangência da disciplina Teoria de Marketing: na primeira, circunscrita em vermelho, está a amplitude desejada da disciplina, abrangendo todos os campos que compõem as relações dos sistemas de marketing; na segunda, circunscrita em azul, está representada a condição atual da baixa abrangência da disciplina, fator preponderante para o distanciamento 
relatado nos artigos especializados. Na ilustração, o campo de interpretação do conhecimento, por possuir acadêmicos e praticantes e uma literatura híbrida, está dividido em dois hemisférios epistemológicos (Smith et al., 2015). Ele é parcialmente atendido pelas teorias de marketing. Já o campo de aplicação do conhecimento é totalmente composto por praticantes e possui uma literatura própria (formada por sites, blogs, revistas não acadêmicas, manuais etc.), bem como um modo próprio de compreender e interferir junto aos problemas, sendo assim o campo mais distante da teoria produzida na academia.

Resumindo esta parte do problema, na busca pelas competências essenciais para a formação em marketing, a literatura investigada até aqui aponta para os seguintes fatos:

1) Devemos considerar que as reivindicações por competências e habilidades em marketing não são exclusivas do mercado; atingem, por exemplo, áreas de marketing social, marketing público e suas múltiplas atuações instrumentais para a solução de problemas acadêmicos, sociais e de mercado (Layton, 1985; 2007; Ferrell \& Ferrell, 2008; Costa, 2015; Kennedy, 2016; Rajan \& Read, 2016; Murphy, 2017; Kadirov, 2018);

2) O problema não é apenas de metodologia de ensino e pesquisa, mas de conteúdo. Os conteúdos de educação em marketing devem ser abrangentes o suficiente para contemplar as demandas dos múltiplos campos de aplicação (Smith et al., 2015; El-Ansary, Shaw \& Lazer, 2018; Rapsold \& Hemais, 2018);

3) Há dúvidas sobre a eficiência da formação atual em marketing, visto que a diplomação - pelo menos, nos contextos pesquisados por Hunt, Chonko e Wood (1986) e Bacon (2017) - não tem surtido efeito sobre a ocupação de cargos no mercado de trabalho;

4) As teorias de marketing estão ainda, em sua maioria, restritas ao ambiente acadêmico (Brown, 2001; Smith et al., 2015; El-Ansary, Shaw \& LAZER, 2018; Rapsold \& Hemais, 2018);

5) No entanto, existe uma influência parcial das teorias de marketing sobre o campo gerencial, especificamente, no nível de interpretação do conhecimento ou de tomada de decisões (Kumar, 2017); esses dois campos ainda apresentam fortes diferenças epistemológicas (Smith et al., 2015).

À guisa de outra visão, tudo que se apresentou até agora nos faz crer que a evolução do campo acadêmico deve acompanhar o progresso do campo gerencial e vice-versa, conferindo aos profissionais formados uma visão amadurecida que permeie tanto a ciência quanto a atuação profissional (com ou sem fins lucrativos) e que seja capaz de impactar de maneira positiva, contínua e significativa as articulações entre os dois campos. Assim, seguimos o entendimento de que os problemas arrolados dizem muito acerca das competências necessárias ao profissional de marketing. É nesse sentido que consideramos que o sujeito doutor é peça chave no desenvolvimento e difusão das teorias científicas e instrumento essencial na formação dos egressos em todos os níveis acadêmicos. Cabe-nos, portanto, uma reflexão sobre a estrutura teórica de formação para que os egressos das linhas de marketing dos programas de doutorado cumpram o seu papel no sentido de integrar conhecimentos gerenciais e acadêmicos em favor do desenvolvimento da ciência e da sociedade (Gokhberg, Meissner \& Shmatko, 2017).

\section{A natureza do marketing e sua relevância para uma educação abrangente}

O termo 'natureza' é bastante utilizado na literatura de teoria de marketing (Bartels, 1974; ElAnsary, Shaw \& Lazer, 2017; Hunt, 1976, 1983, 2002, 2003, 2010; Kotler \& Levy, 1969; Kotler \& Zaltman, 1971; Rajan \& Read, 2016; Shaw, 2014). Mas, seria pouco apropriado entender natureza como sinônimo de conceitoii. Esse é um termo filosófico que se refere à totalidade das característicasiii distintivas de um objeto. Desde a Antiguidade Clássica até os nossos dias, a discussão sobre a natureza das coisas se revela uma importante base epistemológica para a estruturação do conhecimento (Platão, 1997; Aristóteles, 2012; Hegel, 1992, 1995; Kant, 2008; Heidegger, 2009).

A título de exemplo, Hunt $(1976,1983,2000)$ desenvolveu uma linha de pensamento acerca da natureza do marketing considerando as seguintes questões: o que é marketing? Que tipo de fenômeno é apropriadamente denominado fenômeno de marketing? Como atividades de marketing diferem de atividades de não-marketing? 0 que é um sistema de marketing? Como os processos de marketing podem ser distintos de outros processos sociais? Que instituições devem ser referidas como instituições de marketing? Em resumo, qual é o domínio conceitual apropriado ao construto intitulado marketing? Questões desse tipo buscam reunir a totalidade das características e normas relativas ao marketing nas diversas e possíveis manifestações que fluem nas experiências acadêmica, social e profissional. 
Embora, no exemplo acima, Hunt $(1976,1983,2000)$ tenha ofertado a sua contribuição sobre essas questões, as manifestações de marketing ocorrem através de arranjos entre recursos, instituições, agentes e pensamento de marketing - que variam contextualmente, por exemplo, ao longo das " 4 eras" do pensamento de marketingiv (HUNT, 2018a; 2018b). Esses arranjos se ajustam em torno de fatores menores, como recursos físicos, financeiros, metodológicos; intercâmbio com outras disciplinas, normas sociais, legislações, responsabilidade social; tecnologias de transporte, aprendizagem, análise de dados, dentre outros e, simultaneamente, na dependência de um tema dominante que varia com o tempo, assim como os demais fatores.

Na Tabela 1, baseada no trabalho de Kumar (2015), observamos que cada época possui o seu tema dominante e sua própria visão de marketing, influenciada por uma motivação ligada os desafios encontrados pelos profissionais e acadêmicos. É interessante perceber como os recursos, o ambiente (instituições), os agentes e o pensamento dominante de marketing se organizam a partir das interações de cada época, ampliando a atuação de marketing e exigindo reformulações periódicas do seu conceito e, talvez, da compreensão da sua natureza (Hunt, 2018a).

Tabela 1 - Variações da ênfase de marketing ao longo do tempo.

\begin{tabular}{|c|c|c|c|}
\hline Época & Tema dominante & $\begin{array}{l}\text { Visão de mar- } \\
\text { keting }\end{array}$ & Motivações dos temas \\
\hline $\begin{array}{l}1936- \\
1945\end{array}$ & $\begin{array}{c}\text { Princípios e conceitos } \\
\text { de marketing }\end{array}$ & $\begin{array}{l}\text { Economia apli- } \\
\text { cada }\end{array}$ & $\begin{array}{l}\text { - Entender os princípios de marketing através de estudos } \\
\text { de caso; } \\
\text { - Necessidade de entender as legislações governamentais e } \\
\text { as regulações do mercado; } \\
\text { - Tópicos da pesquisa de marketing e implicações práticas. }\end{array}$ \\
\hline $\begin{array}{l}1946- \\
1955\end{array}$ & $\begin{array}{l}\text { Melhoria das funções } \\
\text { de marketing e produ- } \\
\text { tividade dos sistemas }\end{array}$ & $\begin{array}{l}\text { Atividade geren- } \\
\text { cial }\end{array}$ & $\begin{array}{l}\text { - Prestação de contas para a teoria de marketing; } \\
\text { - Foco no crescimento da disciplina; } \\
\text { - Identificação das funções e conceitos de marketing. }\end{array}$ \\
\hline $\begin{array}{l}1950- \\
1965\end{array}$ & $\begin{array}{l}\text { Inclusão dos proble- } \\
\text { mas sociais no escopo } \\
\text { de marketing }{ }^{1}\end{array}$ & Atividade social & $\begin{array}{l}\text { - Utilização do mix de marketing para causas sociais, sem } \\
\text { fins lucrativos; } \\
\text { - Envolvimento com saúde pública; } \\
\text { - Questões humanitárias. }\end{array}$ \\
\hline $\begin{array}{l}1956- \\
1965\end{array}$ & $\begin{array}{l}\text { Avaliação do mix de } \\
\text { mercado }\end{array}$ & $\begin{array}{l}\text { Ciência quanti- } \\
\text { tativa }\end{array}$ & $\begin{array}{l}\text { - Foco no desenvolvimento da teoria de marketing; } \\
\text { - Transição para estudos analíticos; } \\
\text { - Controle estatístico da qualidade. }\end{array}$ \\
\hline $\begin{array}{l}1966- \\
1975\end{array}$ & $\begin{array}{l}\text { Clientes e processos } \\
\text { organizacionais }\end{array}$ & $\begin{array}{l}\text { Ciência compor- } \\
\text { tamental }\end{array}$ & $\begin{array}{l}\text { - Necessidade uma agenda para a disciplina; } \\
\text { - Artigos destacando direções para futuras pesquisas; } \\
\text { - Atenção voltada às práticas de marketing. }\end{array}$ \\
\hline $\begin{array}{l}1976- \\
1985\end{array}$ & $\begin{array}{c}\text { Elaboração de estraté- } \\
\text { gias }\end{array}$ & $\begin{array}{l}\text { Ciência de deci- } \\
\text { são }\end{array}$ & $\begin{array}{l}\text { - Ênfase no desenvolvimento teórico e no entendimento das } \\
\text { estruturas de marketing; } \\
\text { - Perspectiva para o uso das técnicas quantitativas em mar- } \\
\text { keting. }\end{array}$ \\
\hline $\begin{array}{l}1986- \\
1995\end{array}$ & $\begin{array}{l}\text { Identificação de con- } \\
\text { tingências }\end{array}$ & $\begin{array}{c}\text { Ciência integra- } \\
\text { tiva }\end{array}$ & $\begin{array}{l}\text { - Uso de sofisticadas técnicas empíricas; } \\
\text { - Emergência de estruturas conceituais de fenômenos de } \\
\text { marketing; } \\
\text { - Uso de conhecimentos interdisciplinares. }\end{array}$ \\
\hline $\begin{array}{l}1996- \\
2004\end{array}$ & $\begin{array}{l}\text { Lucratividade do cli- } \\
\text { ente e alocação de re- } \\
\text { cursos }\end{array}$ & $\begin{array}{l}\text { Ciência da escas- } \\
\text { sez de recursos }\end{array}$ & $\begin{array}{l}\text { - Desenvolvimento de base de dados tecnológicas; } \\
\text { - Habilidades para capturar dados de clientes individuais; } \\
\text { - Performance de análise no nível individual. }\end{array}$ \\
\hline $\begin{array}{l}2005- \\
2012\end{array}$ & $\begin{array}{l}\text { Responsabilidade de } \\
\text { marketing e foco no } \\
\text { cliente }\end{array}$ & Investimento & $\begin{array}{l}\text { - Avanços tecnológicos; } \\
\text { - Cocriação com o cliente; } \\
\text { - Investimentos em marketing direcionados ao cliente. }\end{array}$ \\
\hline 2013- & $\begin{array}{l}\text { Centralidade do mar- } \\
\text { keting e influência das } \\
\text { mídias }\end{array}$ & $\begin{array}{l}\text { Parte integral } \\
\text { das organiza- } \\
\text { ções }\end{array}$ & $\begin{array}{l}\text { - Mudanças no uso padrão das mídias; } \\
\text { - Foco na eficiência e efetividade de marketing; } \\
\text { - Geração de valor da firma por meio do engajamento dos } \\
\text { stakeholders. }\end{array}$ \\
\hline
\end{tabular}

1'Inserção nossa.

Fonte: Adaptado de Kumar (2015).

Na trajetória desses arranjos, queremos destacar três importantes marcos do pensamento de marketing que influenciaram a resposta acerca da questão sobre a sua natureza: o artigo de Wiebe (1951), intitulado Merchandisign commodities and citizenship on television; o artigo seminal de Kotler e Levy (1969), intitulado Broadening the concept of marketing; e a última definição de marketing estabelecida pela American Marketing Associantion (AMA) em 2007, validada em 2013. 
No início dos anos 1950, com a provocativa pergunta "por que não se pode vender fraternidade e pensamento racional como se vende sabão?"v, Wiebe (1951, p. 679) abriu uma importante discussão sobre a abrangência do marketing e a sua natureza. Seriam fenômenos da categoria de "fraternidade" e "pensamento racional" objetos do escopo de marketing? Quais seriam as implicações desse tipo de inclusão? A proposta de Wiebe (1951) era revolucionária, mas só encontrou respaldo acadêmico quase duas décadas depois, no artigo de Kotler e Levy (1969), transformando a lógica sob a qual as expectativas de marketing eram construídas, abrindo novos caminhos de compreensão da sua natureza. Na publicação de Kotler e Levy (1969), encontramos a ampliação do conceito de marketing, asseverando que o marketing não deveria ser entendido apenas como uma atividade de negócios, mas sim como uma atividade social generalizada.

No segundo artigo dessa importante revolução conceitual sobre o marketing e sua natureza, Kotler e Zaltman (1971) ampliaram não apenas os fenômenos de marketing, como também os seus agentes. Assim, o marketing não deveria mais ser visto apenas como relações entre agentes de mercado, como vendedores e compradores, mas entre quaisquer agentes envolvidos em uma relação de troca com valores econômicos. Fox e Kotler (1980) afirmaram que o marketing estaria preocupado em como as transações são criadas, estimuladas, facilitadas e valoradas. Logo, trata-se de uma ciência que se transforma ao longo do tempo.

Essa discussão tinha como ponto crucial a busca pela identificação dos fenômenos que pudessem ser definidos - a despeito de qualquer arranjo entre recursos, instituições e agentes/pensamentos como pertencentes ao campo do marketing. Parece-nos, então, que a questão está bem definida, do ponto de vista do conceito dominante e oriundo da posição kotleriana, que os fenômenos apropriadamente tipificados como fenômenos de marketing são as "trocas", em suas múltiplas formas (Shaw, 2014; Levy, 2018).

Em julho de 2013, a American Marketing Association (AMA) validou, depois de um longo debate (Costa, 2015), o conceito de marketing predominante no meio científico na atualidade: "Marketing é a atividade, conjunto de instituições e processos para criar, comunicar, entregar e trocar ofertas que têm valor para consumidores, clientes, parceiros e a sociedade em geral"vi (AMA, 2007). Consideramos que a elaboração desse conceito seja o terceiro marco para essa discussão sobre a natureza de marketing por causa da sua tentativa de estabelecer uma definição suficientemente abrangente para incorporar as reivindicações de ampliação do escopo (Kotler \& Levy, 1969), a incorporação de novos agentes (Kotler \& Zaltman, 1971), os pleitos do marketing social derivados do trabalho de Wiebe (1951) e do mainstream de macromarketing (Layton, 2007) na inclusão de trocas não financeiras.

Figura 2 - Desmembramento do conceito de marketing da AMA.

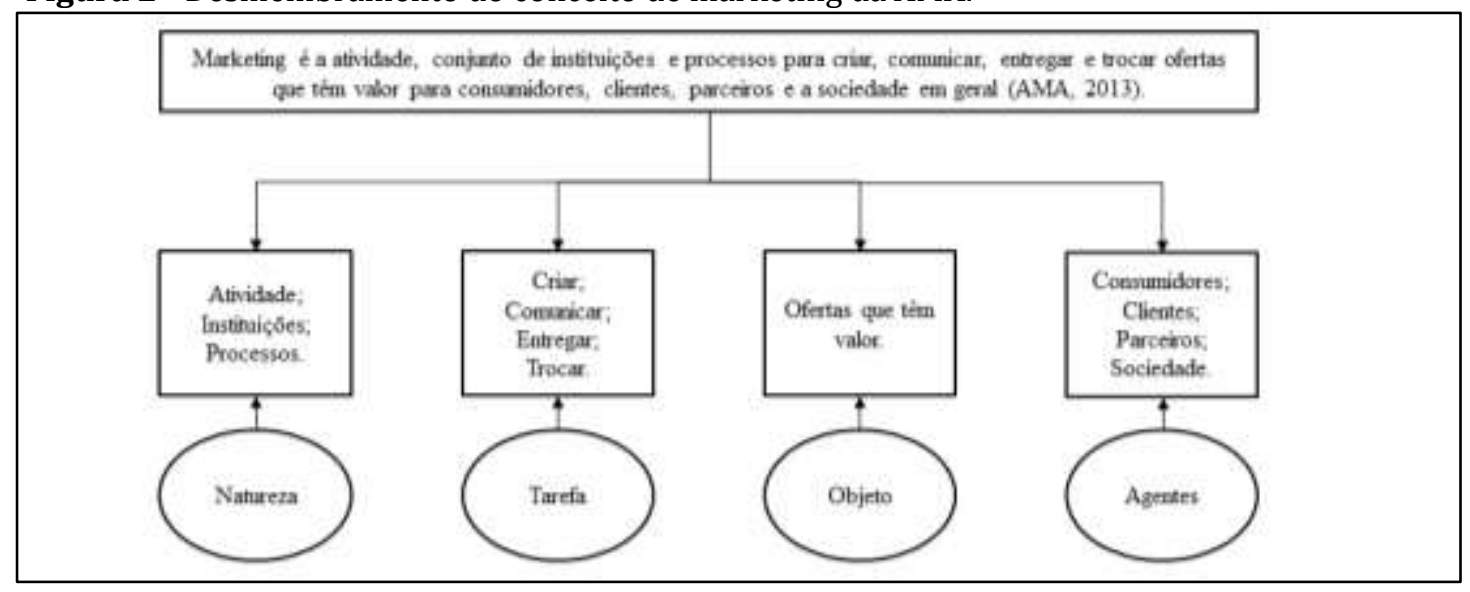

Fonte: Elaboração própria (2020).

Ao definir marketing como "atividade, conjunto de instituições e processos", a AMA (2007) incorporou à natureza do marketing a sua capacidade de fluidez e movimento - não apenas a fluidez do seu conceito (o que seria irrelevante na busca pela natureza do objeto), mas aquela fluidez própria do seu campo de atuação. Esse conjunto de três pequenas expressões define com clareza suficiente as características da "ciência das relações de trocas". Sendo atividade, o marketing é ação dinâmica (Bartels, 1968; Shaw, 2014); sendo conjunto de instituições, o marketing é personificado nos seus agentes e pensamento dominante (Kumar, 2015; Hunt, 2018a); sendo processo, o marketing é uma tecnologia aplicável 
aos diversos campos (Layton, 2007).

Para Bartels (1968), a natureza de marketing estaria definida no seu conceito como função do tempo e do espaço em que sua composição ocorre. Embora Shaw (2014), de certa maneira, reafirme essa posição, dizendo que a natureza de marketing é dinâmica, ele incorpora a essa enunciação de natureza o fato de que a essência do marketing está na existência das transações de mercado que acontecem repetidamente entre as partes atuantes. Se submetermos a ideia de Shaw (2014) às vindicações dos teóricos que desejam que o marketing atue de maneira ampla, teríamos que expandir o conceito de mercado, entendendo-o como um processo exercido pela interação das ações dos vários indivíduos que cooperam na divisão do trabalho (Mises, 2009).

A natureza do marketing, então, vai se desenhando em torno desses marcos - abrangência do objeto (Wiebe, 1951; Kotler \& Levy; 1969), expansão dos agentes (Kotler \& Zaltman, 1971) e desenvolvimento do conceito (AMA, 2007). Mas, haveria uma característica comum a todas as épocas do desenvolvimento do marketing? No final do século XIX, Carl Menger (1840-1921), William Stanley Jevons (18351882) e Frenchman Leon Walras apresentaram ao mundo a teoria do valor marginal (Hunt, 2000). Segundo essa teoria, o preço final dos produtos não é determinado apenas pelos custos de produção, como defendem economistas clássicos, mas também pela agregação de valores subjetivos advindos da relação oferta/demanda. A característica geral do marketing estaria, portanto, definida pela forma como as atividades, instituições e processos de marketing se organizam em torno das ações dos agentes nas atividades que geram ofertas de valor (Alderson, 1937, 1948; Kirkpatrick, 1983, 1985; Hunt, 2000).

Independentemente da época, do pensamento dominante, do arranjo das instituições e da motivação, o marketing visa ao aumento da eficiência e ao incremento do valor marginal para os envolvidos ou interessados na ação de troca. $E$ isso constitui a sua natureza, que é essencialmente empreendedora. A ação empreendedora, por sua vez, está fundamentada nas relações da dinâmica de competição, no estado de alerta e na teoria da ação humana no que tange à promoção das relações de troca visando ao alcance de benefícios mútuos, tal como o lucro decorrente do serviço ou produto que atende às necessidades de outro indivíduo ou conjunto de indivíduos (Kirzner, 2112a).

Para Mises (2009), o processo de mercado é o ajuste das ações individuais dos vários membros da sociedade de mercado para contemplar as necessidades de cooperação mútua. Assim, a título de ilustração, o consumidor, não sendo onisciente, não sabe onde encontrar as melhores ofertas e muito frequentemente não sabe que tipo de mercadoria/serviço pode atender às suas reais necessidades. É, portanto, o propósito do marketing construir o elo entre ofertantes e demandantes da estrutura social, respeitando e cooperando com as regras e valores praticados nessa estrutura. A natureza do marketing, portanto, não estaria definida apenas conceitualmente em um arranjo dos seus componentes, mas sim na sua tarefa primordial de trazer eficiência - e na ideia de eficiência, incluamos construtos da categoria de equilíbrio, justiça etc. - nas relações de troca, buscando a geração de novas oportunidades para se angariar benefícios mútuos entre os componentes de um sistema agregado de marketing.

A próxima questão a ser definida é, portanto, a forma como a atividade de marketing é percebida pelos seus agentes dentro dos contextos acadêmico e não-acadêmico.

\section{Lacunas entre teoria e práticas de marketing: a questão da relevância}

Relevância é um termo que exige complemento, ou melhor, um posicionamento do seu significado dentro do contexto. Dessa forma, a partir da pergunta "relevância para quem?", somos forçados a incorporar um problema mais profundo: se admitíssemos simplesmente que as pesquisas realizadas no âmbito acadêmico são relevantes para a academia e que os conteúdos produzidos no campo profissional são relevantes para o mercado, não estaríamos corroborando a já criticada fragmentação da teoria de marketing e ampliando o distanciamento entre os dois campos? Ainda mais, não estaríamos ampliando as dificuldades para a formulação de uma TGM ou, no mínimo, dificultando a criação de um domínio amplo para a teoria de marketing? E como resultado da ampliação desses dois problemas, não estaríamos dificultando os caminhos para a formação de profissionais e acadêmicos?

Quando Shaw (2014) faz ressurgir a hipótese levantada por Sheth et al. (1988) de que o marketing (como ciência) estaria vivendo uma crise de credibilidade, ele nos leva a retomar uma reflexão que não está ligada apenas à imagem do marketing acadêmico junto ao mercado e à sociedade como um todo, mas, principalmente, sobre a utilidade do campo acadêmico para o mundo real. Essa questão, que já vem de um longo debate, continua presente na atualidade acadêmica, produzindo reflexões profundas sobre o futuro do marketing (Piercey, 1995, 2002; Simkin, 2002; Brennan, 2004; Blythe, 2006; Stringfellow et 
al., 2006; Brownlie, Hewer \& Ferguson, 2007; Ramocki, 2007a; 2007b; Tapp \& Hughes, 2008; Egan, 2009; Jaworski, 2011; Kumar, 2015, 2017).

De maneira geral, o futuro do marketing está vinculado à relevância da teoria de marketing, considerando duas etapas: a) análise do que seria relevante para os setores que utilizam marketing fora da academia; b) reflexões acerca da relevância da teoria de marketing.

\subsection{Relevância da teoria de marketing para as práticas de marketing}

Robstein, Day e Wind (2009), em um editorial do Journal of Marketing, debruçam-se sobre reflexões acerca da existência de uma alarmante e crescente lacuna entre os interesses, os padrões e as prioridades dos acadêmicos e as necessidades dos executivos de marketing. Para eles, essas diferenças existem porque os praticantes operam em um espaço de mercado ambíguo, incerto, complexo e de rápida mudança, ao contrário do ambiente acadêmico, que é programado, rigoroso e opera em ritmo próprio (Mascarenhas, Zambaldi \& Moraes, 2011; Smith et al., 2015). Jaworski (2011), por outro lado, defendia que o problema não estaria no ambiente ou na forma como se define o que é ou não relevante, mas nos atores dessa escolha e em suas perspectivas em relação aos conteúdos que consomem. Ele destaca que: 1) a relevância gerencial é uma avaliação de um gerente em uma função específica; 2) existem múltiplas categorias de relevância gerencial; 3) gerentes consomem muitos tipos de conhecimento gerencial. Assim, a relevância seria relativa e abstrata, definida em função das perspectivas individuais de cada gerente consumidor de conteúdos de marketing, afunilando em direção aos interesses específicos das funções ocupadas pelos demandantes de tais conteúdos. Isso tornaria a produção científica refém de um conjunto imensurável de perspectivas e expectativas particularizadas e das suas dinâmicas pouco previsíveis, transformando a relação entre academia e mercado em um problema fluido e praticamente insanável.

Em um sentido contrário, ao analisar o tema, Dibb, Simões e Wensley (2014) afirmam existir consistência nas visões de acadêmicos e profissionais em relação aos seguintes elementos desagregados da prática: marketing de stakeholders e de relacionamento, análise de clientes, gerenciamento de mix de marketing, planejamento de marketing e centralidade dos clientes. No entanto, quando esses temas são integrados em categorias mais amplas de prática, as atividades são priorizadas de diferentes maneiras pelas diferentes fontes de dados. Certamente, essas diferenças de perspectivas e de fontes de dados são possíveis chaves para a compreensão do distanciamento epistemológico que Smith et al. (2015) compreendem existir entre os dois campos.

À luz das diferenças de perspectivas entre teóricos e praticantes de marketing, encontramos, na literatura, quatro explicações para o apartamento entre teoria e prática: 1) Hill (2001) e Simkin (2002) já destacavam que os currículos de marketing são excessivamente vinculados à estratégia, mas que os praticantes agem, a maior parte do tempo, em nível tático; 2) Egan (2009) afirma que as estratégias que aparecem como temas predominantes nos trabalhos científicos de marketing não são testadas e por esta razão haveria grande dificuldade em converter teoria em prática; 3) a fragmentação do marketing, tanto do ponto de vista teórico quanto do ponto de vista gerencial (Gummesson, 2002; Smith, 2006; Shaw, 2014; Smith et al., 2015; El-Ansary, Shaw \& Lazer, 2018), reduz o potencial de relevância das teorias de marketing para o campo de aplicação; e 4) segundo Blythe (2006), a lentidão do marketing acadêmico é outro fator de afastamento entre teóricos e praticantes. Para ele, as teorias de marketing ainda são ligadas às propostas desenvolvidas nos anos $1960 \mathrm{e}$, mais atualmente, essas mesmas teorias são desafiadas por novas necessidades, como o comércio eletrônico, os novos compostos logísticos, questões sociais de reconhecimento de grupos relevantes para o mercado etc.

Embora possamos defender como justa a ideia de que um campo não está submisso ao outro, precisamos reconhecer que o amplo desenvolvimento de uma TGM depende da cooperação mútua e também de um reposicionamento epistemológico que possibilite os diálogos. Por outro lado, no campo das expectativas, é necessário reconhecer, sem prejuízo das visões mais amplas acerca do conceito e da identidade de marketing, que o marketing foi originalmente uma ferramenta desenvolvida para a realização de negócios (sejam eles com ou sem fins lucrativos), que gerou uma disciplina que promoveu seu desenvolvimento e seus conceitos mais amplos (Alderson, 1948; Hunt, 2010), cabendo, por conseguinte, o necessário reconhecimento por parte dos acadêmicos de que a maior vocação do marketing está relacionada ao campo da prática profissional e da geração de negócios. Essa expectativa, obviamente, afeta não apenas as abordagens epistemológicas, como também as estruturas metodológicas e as fontes de dados utilizadas no cotidiano do marketing (Ruiz \& Holmlund, 2017). 
A relevância do marketing, portanto, será sempre medida pela sua capacidade de atender aos seus dois grandes públicos (acadêmicos e praticantes). Então, é necessário que a academia formule uma agenda de pesquisas que inclua a possibilidade das ações práticas do marketing no mercado e na sociedade em temas que permeiam as principais preocupações sociais, tais como a contribuição do marketing para o sistema financeiro do mercado global (Papadopoulos \& Heslop, 2014; Crownder \& Reganold, 2015); as questões mais importantes de políticas públicas, tais como drogas, obesidade, violência no trânsito, álcool (Niland et al., 2018); desenvolvimento regional e marketing de locais, turismo e regionalidade (Lichrou, O'malley \& Patterson, 2014; Abdelhadi; Forster \& Whysall, 2014; Seabra, Abrantes \& Kastenholz, 2014), além de muitos outros temas e gêneros alcançáveis pelo conceito expandido de marketing. Isso permitiria alcançar crescimento lucrativo, encontrando, através das melhores práticas de marketing, os caminhos mais viáveis para a inovação, de modo a criar novo valor para os clientes e, simultaneamente, contornando os riscos que lhe são próprios, além de usar os recursos dos parceiros em inovações de modelos abertos e assim por diante (Robstein, Day \& Wind, 2009; Kumar, 2015, 2017), dentre outros possíveis interesses comuns, tais como alocação dinâmica de recurso, proliferação de novos canais de comunicação e distribuição, mudanças no poder político do consumidor etc.

\subsection{Reflexões acerca da relevância da teoria de marketing}

As práticas de marketing, tanto do ponto de vista empresarial quando dos pontos de vista social e acadêmico, possuem uma larga gama de questões que fazem parte do circuito de debates nos congressos e periódicos. Por exemplo, ambientes e condições de crescimento empresarial (Zhu \& Matsuno, 2016); eficácia em ambientes de vulnerabilidade e risco social (Miles et al., 2016); novas tecnologias e suas aplicações (Ahmadi \& O'cass, 2016); políticas públicas e decisões de empresas sem fins lucrativos e de governos (Wilkie \& Gardner, 1974; Hunt; Morgan, 1995); impactos das estratégias de marketing sobre as pessoas (Olson et al., 2018), dentre muitos outros não apresentados aqui. No entanto, a transposição entre os campos acadêmico e gerencial ainda é pequena ou quase inexistente. Em outras palavras, a consistência defendida por Dibb, Simões e Wensley (2014) não é suficiente para que os gerentes, em geral, modifiquem a sua estrutura epistemológica ou os seus processos decisórios, nem mesmo os seus ritmos de atuação. A despeito de todos os esforços acadêmicos, o mundo gerencial continua fluindo em uma velocidade diferente e em um ambiente mais improvisado, distante do framework acadêmico.

Diante da permanência do impasse, Robstein, Day e Wind (2009) sugerem que os pesquisadores de marketing devem construir, em colaboração com os gerentes, uma nova agenda de pesquisas que possa oferecer, dentro dos campos de interesse, perspectivas amplas ou significações pontuais que façam parte de um contexto plausível de prática de marketing. Os autores esperam, portanto, que o acadêmico de marketing esteja capacitado para, junto ao mercado, discutir questões críticas de marketing estratégico e/ou questões emergentes, tais como o impacto das organizações em rede, o valor da inovação aberta, cadeias de valor, práticas antiéticas de marketing, o papel das marcas em ambientes globais, enfim, problemas mais práticos e rotineiros do marketing aplicado ao cotidiano das organizações e da sociedade.

Sugestões como as de Robstein, Day e Wind (2009) parecem plenamente factíveis, mas deixam uma dúvida: por que ainda não repercutiram de maneira positiva no aumento da relevância acadêmica para o mercado? As respostas a essas questões são buscadas em muitos trabalhos da área (Diziubaniuk et al., 2020; Koskull, 2020; Zeithaml et al., 2020). 0 fato é que a discussão entre rigor e relevância, na forma como tem sido conduzida, tem se revelado distópica. Trata-se da tentativa de unir elementos imiscíveis na ordem prática, pois ignoram os elementos mais profundos da ação humana que guiam a problemática de cada área na qual o marketing atua. Os problemas de marketing são oriundos da realidade existencial e raramente se repetem da mesma forma como fenômenos da Física ou da Química que podem ser isolados em laboratórios. Por isso, é improvável que, como deseja Hunt (2002), tenhamos um conjunto de leis do tipo se-então para a formação de uma TGM.

Uma lacuna clara aparece nas propostas para a aproximação entre academia e mercado: é a falta de uma antropologia clara que identifique o marketing como ação social que depende da ação humana. Como ciência social, o marketing tem foco naquilo que se entrega ou recebe do agente humano que ocupa diferentes posições no mercado ou em outros campos das práticas de marketing. É nesse sentido que o trabalho de Mises (2009) defende que a ação humana, para quaisquer posições, é uma ação via de regra empreendedora, pois todo ser humano busca, na vida social, oportunidades para atender aos seus interesses ou de outrem (Kirzner, 2016). Em suma, o marketing que se origina na vida cotidiana visa ao 
atendimento das necessidades empreendedoras, buscando eficiência para a solução de problemas reais. Propomos uma reflexão no sentido de construir uma relação mais profunda do marketing com a ação empreendedora. Esses temas estão, em nosso entender, bastante vinculados a um conceito amplo de empreendedorismo, definido nos termos de Kirzner $(2011,2012)$ como uma busca por oportunidades dentro de um ambiente social. Nesse sentido, é possível defender a visão de que o empreendedorismo é, senão o principal, um dos principais caminhos para a integração entre os interesses acadêmicos e profissionais que podem oferecer relevância à teoria de marketing. Na teoria kirzneriana, o empreendedorismo não está necessariamente relacionado à inovação, mas à ocupação de lacunas no mercado, não apenas criando um novo valor, mas entregando valor, não necessariamente novo, ao mercado.

Embora os teóricos neoclássicos se concentrem no equilíbrio, com uma noção estática da natureza da competição, os economistas da escola austríaca consideram os mercados como processos de descoberta que mobilizam informações dispersas (Jacobson, 1992; Kirzner, 2012; Mises, 2012). Os pensadores da escola austríaca afirmam que as empresas obtêm lucros por meio da descoberta empresarial e concentram-se no empreendedor, motivados pelo desejo de lucros supranormais, como um veículo para promover a descoberta e para a realização de oportunidades em um mercado em constante mudança, isto é, em estado permanente de desequilíbrio (Kirzner, 1997, 1999, 2010, 2012). Como os concorrentes imitam estratégias conhecidas por gerar retornos acima do normal até que o prêmio de retorno seja eliminado, os retornos anormais associados a essa descoberta são apenas temporários.

A ação de marketing, nesse contexto, deve, segundo Whalen e Akaka (2016), considerar a cocriação de oportunidades como um construto fundamental para o avanço da disciplina de marketing especificamente ligada ao empreendedorismo, integrando visões emergentes do empreendedorismo com uma lógica de marketing em evolução - como a lógica dominante de serviços - para desenvolver uma conceituação de mercado capaz de explorar a incerteza em ambientes sociais e econômicos dinâmicos. Ainda segundo Whalen e Akaka (2016), com essa conceituação de mercado, as oportunidades são continuamente cocriadas através do desenvolvimento e comunicação de proposições de valor, da derivação e determinação de valor e da (re)formação de mercados. Seguindo esse caminho, pesquisas emergentes em torno do processo de cocriação de oportunidades poderiam ser usadas para criar uma definição inicial e considerações teóricas em marketing.

\section{Considerações finais}

A disciplina Teoria de Marketing não tem uma relação tautológica com a TGM, visto que essa última é, por natureza, maior do que a primeira. No entanto, uma vez definida, a TGM deve possuir um espaço para a sua evolução e difusão. Afinal, a ciência amadurece à medida que os desenvolvimentos teóricos melhoram por meio dos debates ao longo do tempo, contribuindo para o corpo do pensamento histórico e para o seu enriquecimento. À medida que o ciclo pensamento-teoria continua a se regenerar, a ciência social do marketing continua a progredir (Shaw, 2011).

Defendemos, neste trabalho, que o espaço acadêmico para este fim é a disciplina Teoria de Marketing, na qual um conjunto de temas basilares deve ser organizado e debatido com/por acadêmicos de todos campos do marketing. Costa (2015, p. 11) afirma que "marketing é uma disciplina acadêmica e profissional". Nesse sentido, podemos inferir que uma TGM deve dar conta das duas carreiras, oferecendo uma visão ampla o suficiente para responder às questões ontológicas, promovidas no ambiente interno da academia, e às questões práticas, originárias da aplicação do marketing enquanto tecnologia e seus instrumentos, tanto no campo gerencial do âmbito empresarial quanto nas aplicações de ordem sociais (Ewing \& Ewing, 2017).

Antes, porém, precisamos conhecer os pressupostos apresentados por Bartels (1968) que funcionam como sedimento para a concepção de uma TGM: 1) marketing é um fenômeno universal com diferentes níveis de sofisticação; 2) o marketing tem sua tarefa e estrutura influenciadas pelas circunstâncias geográficas, econômicas, temporais, informacionais etc., 3) os participantes do marketing agem em sistemas nos quais processam os fluxos necessários e, nesses papéis, atuam como entidades sociais; 4) a estrutura institucional de marketing representa uma divisão econômica do processo de marketing e unidades tecnicamente diferenciadas para fins econômicos; 5) tanto a tecnologia de marketing quanto a ética social guiam as relações de marketing; 6) como um processo culturalmente orientado de interação social, o marketing está subordinado ao objetivo social.

O caráter abrangente de uma TGM, definida nos termos da literatura utilizada (Bartels, 1968; Hunt, 2003; Shaw, 2014; El-Ansary, Shaw \& Lazer, 2018), sugere que todas as teorias do campo devem 
ser abarcadas conjuntamente. Da Tabela 1, em conjunto com os pressupostos de Bartels (1968), foram extraídos cinco grupos de conhecimentos e habilidades técnicas considerados de alta relevância para a composição de uma formação abrangente em teoria de marketing que resumimos na Tabela 2.

Tabela 2 - Conhecimentos sedimentares de Teoria de Marketing.

CONHECIMENTOS

AUTORES

\begin{tabular}{|c|c|}
\hline $\begin{array}{l}\text { O processo de formação do conhecimento no campo específico de } \\
\text { marketing. Metodologias adequadas ao escopo geral da disciplina. }\end{array}$ & $\begin{array}{l}\text { Jones (1905); Shaw (1912); Halbert (1965); Hunt } \\
\text { (1983 2000): November (2002): Kennedy (2017) }\end{array}$ \\
\hline $\begin{array}{l}\text { O marketing enquanto tecnologia de gestão das atividades, institui- } \\
\text { ções e processos nas organizações e na sociedade. }\end{array}$ & $\begin{array}{l}\text { Hunt (1976, 2018a); Kumar (2017); Shaw (2014); } \\
\text { Rapsold \& Hemais (2018). }\end{array}$ \\
\hline $\begin{array}{l}\text { Perspectivas do desenvolvimento do pensamento e da prática em } \\
\text { marketing. Como a ciência se identifica, se define e evolui. }\end{array}$ & $\begin{array}{l}\text { Fullerton (1988); Hunt }(2010) \text {; Kumar (2015); Jones } \\
\& \text { Shaw (2018) Shaw }(2011,2014) .\end{array}$ \\
\hline $\begin{array}{l}0 \text { marketing e o interesse social. As tecnologias e pensamentos de } \\
\text { marketing utilizados no desenvolvimento de atividades e institui- } \\
\text { cões de interesse das sociedades. }\end{array}$ & $\begin{array}{l}\text { Barrios et al. (2016); Costa (2015); Haase, Becker \& } \\
\text { Pick (2017); lyer \& Reczek (2017); Murphy (2017). }\end{array}$ \\
\hline
\end{tabular}

Fonte: Autoria própria (2020).

Diferente de propor um ajuntamento de todas as teorias de marketing, o escopo do nosso projeto é definido pelo agrupamento desses conhecimentos em um ambiente propício para a difusão e desenvolvimento da teoria de marketing, isto é, na disciplina Teoria de Marketing aplicada em nível de doutorado. A nossa proposta é fazer que esses temas, que inicialmente discutimos, sirvam de base para a disciplina Teoria de Marketing, buscando, conforme ilustramos na Tabela 2, incorporar o caráter universal do marketing, as influências circunstanciais, a participação dos agentes de marketing como agentes sociais, as estruturas institucionais, o caráter ético e social e a integração aos designíos sociais como parte consolidadora de uma estrutura de conhecimento para os egressos das linhas de marketing nos doutorados em Administração.

\section{Referências}

Abdelhadi, A., Foster, C., \& Whysall, P. (2014). An exploratory investigation of aberrant consumer behavior in Libya: a sociocultural approach. Journal of Marketing Management, 30(9-10), p. 857-873.

Ahmadi, H., \& O'cass, A. (2016). The role of entrepreneurial marketing in new technology ventures first product commercialization. Journal of Strategic Marketing, 24(1), p. 47-60.

Alderson, W. (1937). A marketing review competition. Journal of Marketing, 1(3), 189-190.

Alderson, W., \& COX, R. (1948). Toward a theory of marketing. Journal of Marketing, 13(2), p. 137-151.

AMA - American Marketing Association (2007). Definition of Marketing of 2007. Disponível em: $<$ http://www.marketingpower.com>. Acesso 14 mar de 2020

Aristóteles. (2012). Metafísica. São Paulo: Edipro.

Bacon, D. R. (2017). Revisiting the relationship between marketing education and marketing career success. Journal of Marketing Education, 39(2), p. 109-123.

Baggozi, R. P. (1975). Social exchange in marketing. Journal of the Academy of Marketing Science, 3(3-4), p. 314-327.

Barrios, A., Valk, K., Shultz, C., Sibai, O., Husemann, K. C., Maxsell-Smith, M., \& Luedicke, M. (2016). Marketing as a means to transformative social conflict resolution: lessons from transitioning war economies and the Colombian coffee marketing system. Journal of Public Policy \& Marketing, 35(2), p. 2-36.

Bartels, R. (1974). The identity crises in marketing. Journal of Marketing, 38(4), p. 73-76.

Bartels, R. (1968). The general theory of marketing. Journal of Marketing, 32(1), p. 29-33.

Blythe, J. (2006). A very short, fairly interesting and reasonably cheap book about studying marketing. London: Sage Publications Ltd.

Brennan, R. (2004). Should we worry about an "academic-practitioner divide"? Marketing Intelligence and Planning, 22(5), p. 492-500.

Bringberg, D., \& Mcgrath, J. E. (1983). External validity and the research process: A comment on the Calder/lynch dialogue. Journal of Consumer Research, 10(1), p. 115-124.

Brown, S. (2001). Art or science?: Fifty years of marketing debate. Journal of Marketing Management, 12(4), p. 243-267.

Brownlie, D., Hewer, P., \& Ferguson, P. (2007). Theory into practice: meditations on cultures of accountability and interdisciplinarity in marketing research. Journal of Marketing Management, 23(5/6), p. 395-409. 
Calder, B. J., Phillips, L. W., \& Tybout, A. M. (1981). Designing research for application. Journal of Consumer Research, 8(2), p. 197-207.

Converse, P. D. (1945). The development of Science of Marketing. Journal of Marketing, 10(1), p. 14-23.

Costa, F. J. (2015). Marketing e sociedade. João Pessoa: Editora UFPB.

Crownder, D. W., \& Reganold, J. P. (2015). Financial competitiveness of organic agriculture on a global scale. PNAS, 112(24), p. 7611-7616.

Dibb, S., Simões, C., \& Wensley, R. (2014). Establishing the scope of marketing practice: insight from practitioners. European Journal of Marketing, 48(1/2), p. 380-404.

Diziubaniuk, O., Rasmussem, W. B., Koporcic, N., Gongne, M. I., Mandjak, T., \& Markovic, S. (2020). Business-to-business marketing research: assessing readability and discussing relevance to practitioners. Journal of Industrial Marketing Management (in press).

Egan, J. (2009). Reflections on the art-science debate. The Marketing Review, 9(1), p. 31-38.

El-Ansary, A., Shaw, E. H., \& Lazer, W. (2018). Marketing's identity crisis: insights from the history of marketing thought. Academy of Marketing Science, 8(1-2), p. 5-17.

Ewing, D. R., \& Ewing, R. L. (2017). Leveraging experiential learning to encourage role transition from "student" to "professional": Insights from identity theory. Journal of Marketing Education, 39(3), p. 132-144.

Ferrell, O. C., \& Ferrrell, L. (2008). A macromarketing ethics framework: stakeholder orientation and distributive justice. Journal of Macromarketing, 28(1), p. 24-32.

Fox, K., \& Kotler, P. (1980). The marketing of social causes: The first 10 years. Journal of Marketing, 44(1), p. 24-33.

Fullerton, R. A. (1988). How modern is modern marketing? Marketing's evolution and the myth of "Production Era". Journal of Marketing, 52(1), p. 108-125.

Gokhberg, L., Meissner, D., \& Shmatko, N. (2017). Myths and realities of highly qualified labor and what it means for PhDs. Journal of the Knowledge Economy, 8(2), p. 758-767.

Gummesson, E. (2002). Relationship marketing and a new economy: it's time for de-programing. Journal of Services Marketing, 16(7), p. 585-589.

Haase, M., Becker, I., \& Pick, D. (2018). Alternative economies as Marketing System? The role of value creation and the criticism of economic growth. Journal of Macromarketing, 38(1), p. 57-72.

Halbert, M. (1965). The meaning and sources of marketing theory. New York: McGraw Hill.

Heidegger, M. (2009). Introdução à Filosofia. 2· ed. São Paulo: Martins Fontes.

Hegel, G. W. F. (1995). Filosofia da história. Brasília-DF: Ed. UnB.

Hegel, G. W. F. (1992). A fenomenologia do espírito. Petrópolis-RJ: Vozes.

Hill, J. (2001). A multidimensional study of the key determinants of effective SME marketing activity: Part 1. International Journal of Entrepreneurial Behavior \& Research, 7 (5), p. 171-204.

Honea, H., Castro, I. A., \& Peter, P. (2017). Evidence items as a signals of marketing competencies and workplace readiness: A practitioner perspective. Journal of Marketing Education, 39(3) p. 145-161.

Hunt, S. D. (2018a). Advancing marketing strategy in the marketing discipline and beyond: from promise, to neglect, to prominence, to fragment (to promise?). Journal of Marketing Management, 34 (1-2), p. 16-51.

Hunt, S. D. (2018b). The prospects for marketing strategy and the marketing discipline in Era V: is the prognosis promising or problematic? Journal of Marketing Management, 34 (1-2), p. 86-95.

Hunt, S. D. (2010). Explorations \& Insights: Doctoral seminars in marketing theory for incorporating the history of marketing practice and thought. Journal of Historical Research in Marketing, 2(4), p. 443-456.

Hunt, S. D. (2003). Controversy in Marketing Theory: For reason, realism, truth and objectivity. Armonk, NY: ME Sharpe.

Hunt, S. D. (2002). Foundations of Marketing Theory: Toward a general theory of marketing. Armonk, NY: ME Sharpe.

Hunt, S. D. (2000). The general theory of competition: Resources, competences, productivity, economic growth. Series: Marketing for a new century. First Edition. London: SAGE Publications.

Hunt, S. D. (1983). General theories and the fundamental explananda of marketing. Journal of Marketing, 47(4), p. 9-17.

Hunt, S. D. (1976). The nature and scope of Marketing. Journal of Marketing, 40 (2), p. 19-24.

Hunt, S. D., Chonko, L. B., \& Wood, V. R. (1986). Marketing education and marketing success: Are they related? Journal of Marketing Education, 8(2), p. 2-13. 
Hunt, S. D., \& Morgan, R. M. (1995). The comparative advantage theory of competition. Journal of Marketing, 59(2), p. 1-15.

Iyer, E. S., \& Reczek, R. W. (2017). The intersection of sustainability, marketing, and public policy: introduction to the special section on sustainability. Journal of Public Policy \& Marketing, 36 (2), p. 246-254.

Jacobson, R. (1992). The "Austrian" School of Strategy. Academy of Management Review, 17(4), p. 782807.

Jaworski, B. J. (2011). On managerial relevance. Journal of Marketing, 75(4), p. 211-224.

Jones, D. G. B., \& Shaw, E. H. (2018). Avoiding academic irrelevance in the marketing discipline: the promise of the history of marketing thought. Journal of Marketing Management, 34(1-2), p. 52-62.

Jones, E. D. (1905). The manufacturer and domestic market. The ANNALS of the American Academy of Political and Social Science, 25(1), p. 1-20.

Kadirov, D. (2018). Toward a theory of marketing system as the public good. Journal of Macromarketing, 38(3), p. 278-297.

Kant, I. (2008). Prolegômenos a toda metafísica futura. Lisboa: Edições 70.

Kennedy, A. M. (2016). Macro-social marketing. Journal of Macromarketing, 36(3), p. 354-365.

Kirkpatrick, J. (1983). Theory and history in marketing. Managerial and Decision Economics, 4(1), p. 4449.

Kirkpatrick, J. (1985). Theory and history in marketing: Reply. Managerial and Decision Economics, 6(3), p. 186-188.

Kirkpatrick, J. (2018). Objectivist epistemology as the foundation of Marketing Theory. In: Younkins, E. W. (Org.). Perspectives on Ayn Rand's contributions to economic and business though. Lexington Books: New York.

Kirzner, I. M. (2016). Discovery, capitalism, and distributive justice. Indianapolis: Liberty Fund.

Kirzner, I. M. (2015). Austrian subjectivism and the emergency of entrepreneurship theory. Indianapolis: Liberty Fund.

Kirzner, I. M. (2012a). Competição e atividade empresarial. 2. ed. São Paulo: Instituto Ludwig von Mises Brasil.

Kirzner, I. M. (2012b). Essays on Capital Interest. Indianapolis: Liberty Fund.

Kirzner, I. M. (2011). Market theory and the price system. Indianapolis: Liberty Fund.

Kirzner, I. M. (1999). Creativity and/or alertness: A reconsideration of the Schumpeterian entrepreneur. Review of Austrian Economics, 11, p. 5-17.

Kirzner, I. M. (1997). Entrepreneurial discovery and the competitive market process: an Austrian approach. Journal of Economic Literature, 35(1), p. 60-85.

Koskull, C. V. (2020). Increasing rigor and relevance in service research through ethnography. Journal of Services Marketing (in press).

Kotler, P., \& Levy, S. J. (1969). Broadening the concept of marketing. Journal of Marketing, 33(1), p. 1015.

Kotler, P., \& Zaltman, G. (1971). Social marketing: An approach to planned social change. Journal of Marketing, 35(3), p. 3-12.

Kumar, V. (2017). Integrating theory and practice in marketing. Journal of Marketing, 81(2), p. 1-7.

Kumar, V. (2015). Evolution of Marketing as a Discipline: What has happened and what to look out for. Journal of Marketing, 79 (1), p. 1-9.

Laczniak, G. R., \& Santos, N. J. C. (2010). The integrative justice model for marketing to the poor: An extension of S-D logic to distributive justice and macromarketing. Journal of Macromarketing, 31(2), p. $135-147$.

Layton, R. (2011). Marketing: is management all that there is? Journal of Historical Research in Marketing, 3(2), p. 194-213.

Layton, R. (2007). Marketing Systems: a core macromarketing concept. Journal of Macromarketing, 27 (3), p. 227-242.

Layton, R. (1985). Marketing system in regional economic development. Journal of Macromarketing, 5 (1), p. 42-55.

Lichrou, M., O'Malley, L., Patterson, M. (2014). On the marketing implications of place narratives. Journal of Marketing Management, 30(9-10), p. 832-856.

Mascarenhas, A. O., Zambaldi, F., \& Moraes, E. A. (2011). Rigor, relevância e desafios da academia em administração: tensões entre pesquisa e formação profissional. Revista de Administração de Empresas, 
51 (3), p. 265-279.

Miles, M. P., Lewis, G. K., Hall-Phillips, A., Morrish, S. C., Gilmore, A., \& Kasouf, C. (2016). The influence of entrepreneurial marketing processes and entrepreneurial self-efficacy on community vulnerability, risk, and resilience. Journal of Strategic Marketing, 24 (1), p. 34-46.

Mises, L. (2009). Ação Humana. São Paulo: Instituto Ludwig von Mises Brasil.

Murphy, P. E. (2017). Research in marketing ethics: Continuing and emerging themes. Recherche et Applications en Marketing, 32(3), p. 84-89.

Niland, P., McCreanor, T., Lyons, A. C., \& Griffin, C. (2017). Alcohol marketing on social media: young adults engage with alcohol marketing on Facebook. Journal of Addiction Research \& Theory, 25(4), p. 273-284.

November, P. (2002). Teaching marketing theory. A hermeneutic approach. Journal of Marketing Theory, 2(1), p. 115-132.

Olson, E. M., Slater, S. F., Hult, G. T. M., \& Olson, K. M. (2018). The application of human resource management policies within the marketing organization: The impact of business and marketing strategy implementation. Industrial Marketing Management, 69, p. 62-73.

Papadopoulos, N., \& Heslop, L. A. (2014). Product-Country images: Impact and role in international marketing. New York: Routledge.

Piercey, N. (2002). Research in marketing: teasing with trivia or risking relevance? European Journal of Marketing, 36(3), p. 350-363.

Piercey, N. (1995). Marketing and strategy fit together (in spite of what some may say). Management Decisions, 33 (1), p. 42-48.

Platão. (1997). A República. São Paulo: Nova Cultural, (Coleção Os Pensadores).

Rajan, K. M., \& Read, S. (2016). Value co-criation: concept and measurement. Journal of the Academics Marketing Science, 44(3), p. 290-315.

Ramocki, S. P. (2007a) A critical challenge awaiting marketing education. Marketing Education Review, $17(3)$, p. 12-20.

Ramocki, S. P. (2007b). Metacognition and transfer: keys to improving marketing education. Journal of Marketing Education, 29(1), p. 18-24.

Rapsold, F. C., \& Hemais, M. W. (2018). Divide in marketing between academics and practitioners. Brazilian Business Review, 15 (1), p. 68-87.

Robstein, D. J., Day, G., \& Wind, J. (2009). Guest Editorial: Is marketing academia losing its way? Journal of Marketing, 73 (4), p. 1-3.

Ruiz, C. D., Holmlund, M. (2017). Actionable marketing knowledge: A close reading of representation, knowledge and action in market research. Industrial Marketing Management, 66, p. 172-180.

Santos, N. J. C., \& Laczniak, G. R. (2009). Marketing to the poor: an integrative justice model for engaging impoverished market. Journal of Public Policy \& Marketing, 28(1), p. 3-15.

Seabra, C., Abrantes, J. L., \& Kastenholz, E. (2014). The influence of terrorism risk perception on purchase involvement and safety concern of international travelers. Journal of Marketing Management, 30(9$10)$, p. 874-903.

Shaw, E. (2014). The quest for a general theory of marketing system. Journal of Historical Research in Marketing, 6(4), p. 523-537.

Shaw, E. (2011). A comment on the relationship between the history of marketing thought and the development of marketing theory. Journal of Marketing Theory, 11(4), p. 491-494.

Sheth, J. N., Gardner, D. M., \& Garrett, D. E. (1988). Marketing theory: Evolution and evaluation. John Wiley \& Sons, Inc., New York.

Simkin, L. (2002). Tackling implementations impediments to marketing. Marketing Intelligence and Planning, 20 (2), p. 120-126.

Smith, T., Williams, T., Lowe, S., Rod, M., \& Hwang, K. S. (2015). Context into text into context: marketing practice into theory; marketing theory into practice. Marketing Intelligence \& Planning, 33(7)p. 10271046.

Stringfellow, L., Ennis, S., Brennan, R., Harker, M. J. (2006). Mind the gap: The relevance of marketing education to marketing practice. Market Intelligence and Planning, 24(3), p. 245-256.

Tapp, A., \& Hughes, R. (2008). Why "soft science" is the key to regaining leadership in marketing knowledge. European Journal of Marketing, 42(3/4), p. 265-278.

Whalen, P. S., Akaka, M. A. (2016). A dynamic market conceptualization for entrepreneurial marketing: 
the co-creation of opportunities. Journal of Strategic Marketing, 24(1), p. 61-75.

Wiebe, G. D. (1951). Merchandising commodities and citizenship on television. Public Opinion Quarterly, 15 (4), p. 679.

Wilkie, W. L., \& Gardner, D. M. (1974). The role of marketing research in public policy decision making: How can marketing research aid the public policy maker? Journal of Marketing, 38 (1), p. 38-47.

Wilkie, W. L. (2007). Explorations of marketing in society. Manson, Thomson Higher Education: Ohio.

Ye, C., Van Os, J., Chapman, D., \& Jacobson, D. (2017). An on-line project-based competence education approach to marketing education. Journal of Marketing Education, 39(3), p. 162-175.

Zeithaml, V. A., Jaworki, B. J., Kohli, A. K., Tuli, K. R., Ulaga, W., \& Zaltman, G. (2020). A theory-in-use approach to building marketing theory. Journal of Marketing, 83(1), p. 32-51.

Zhu, Z., \& Matsuno, K. (2016). Entrepreneurial proclivity: its environmental conditions and growth consequences. Journal of Strategic Marketing, 24(1), p. 20-33.

\footnotetext{
i Uso o termo "gerencial" de maneira bastante ampla, referindo-me a tudo aquilo que não é acadêmico, inclusive às aplicações de macromarketing, marketing social, marketing público etc.

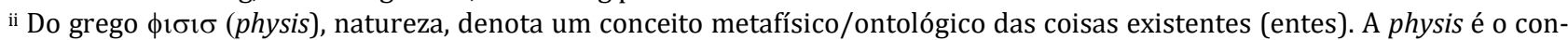
junto de características distintivas da espécie da qual o ente faz parte. Para Platão, a physis é imutável. Para Aristóteles, ela acompanha o fluir das mudanças, mantendo as características distintivas correspondentes à identidade do ente, esta, sim, imutável. Ambos concordam que a natureza trata das características definidoras e distintivas do ente.

iii A expressão utilizada por Kant é "Gesamtheit der Regeln", mais corretamente traduzida como "totalidade das regras" e não das "características" como inserimos no texto. No entanto, a despeito da defesa de que uma tradução literal é, em geral, mais precisa, cremos que a palavra "característica" é hermeneuticamente mais coerente e adequada ao nosso uso, com vistas a evitar uma discussão filosófica sobre sentido de "totalidade das regras".

iv As quatro eras do marketing a que nos referimos são definidas pelo trabalho de Wilkie e Moore (2003) da seguinte forma: A Era I seria a fundação do campo (Founding of field); a Era II seria a formalização do campo (Formalizing of the field); a Era III seria a era do marketing estratégico (Marketing strategy) e a Era IV seria a era do relacionamento (Relationship mangement marketing).

v Tradução livre para: "Why can't you sell brotherhood and rational thinking like you can sell soap?".

vi Tradução livre para: "Marketing is the activity, set of institutions, and processes for creating, communicating, delivering, and exchanging offerings that have value for customers, clients, partners, and society at large".
} 